\title{
Specializations of Ferrers ideals
}

\author{
Alberto Corso • Uwe Nagel
}

Received: 19 March 2007 / Accepted: 30 November 2007 / Published online: 14 December 2007

(C) Springer Science+Business Media, LLC 2007

\begin{abstract}
We introduce a specialization technique in order to study monomial ideals that are generated in degree two by using our earlier results about Ferrers ideals. It allows us to describe explicitly a cellular minimal free resolution of various ideals including any strongly stable and any squarefree strongly stable ideal whose minimal generators have degree two. In particular, this shows that threshold graphs can be obtained as specializations of Ferrers graphs, which explains their similar properties.
\end{abstract}

Keywords Ferrers graphs · Threshold graphs · Monomial (edge) ideals · Cellular minimal free resolution

\section{Introduction}

One of the starting points of this note has been the observation that two very common classes of graphs, namely Ferrers graphs and threshold graphs, have similar properties (see, e.g., [9]). This is remarkable as Ferrers graphs are particular bipartite graphs on vertex sets $\left\{x_{1}, \ldots, x_{n}\right\}$ and $\left\{y_{1}, \ldots, y_{m}\right\}$, whereas threshold graphs are typically not bipartite. One of the goals of this article is to show that the similarity between these graphs extends to algebraic properties of their edge ideals and that it has a natural interpretation. In fact, in [4] we have described a cellular minimal free resolution of Ferrers ideals, the edge ideals of Ferrers graphs. The polyhedral cell complex

\footnotetext{
A. Corso $(\bowtie) \cdot$ U. Nagel

Department of Mathematics, University of Kentucky, Lexington, KY 40506, USA

e-mail: corso@ms.uky.edu

U. Nagel

e-mail: uwenagel@ms.uky.edu

Present address:

U. Nagel

Institute for Mathematics \& Its Applications, University of Minnesota, Minneapolis, MN 55455, USA
} 
that governs this cellular resolution has a very nice geometric description as a certain subcomplex of the face complex of the product of two simplices. This allows us to compute various invariants of Ferrers ideals such as, for example, their $\mathbb{Z}$-graded Betti numbers and their height. The main idea of this paper is that this information can be used to gain insight about graphs that are often not bipartite by using a 'specialization' process (see Section 3). Roughly speaking, specializing simply means to identify each $y$-vertex with an $x$-vertex. Extending this specialization to the polyhedral cell complex that resolves the Ferrers ideal provides, under suitable hypotheses, a cellular minimal free resolution of the specialized Ferrers ideal that is not necessarily a squarefree monomial ideal. After some preliminaries this program is carried out in Section 3. In Section 4 we discuss the class of ideals and graphs that can be described by using specializations of Ferrers ideals. In particular, we show that all threshold graphs can be obtained as specializations of Ferrers graphs. Furthermore, every strongly stable ideal that is generated in degree two can be obtained as such a specialization.

Horwitz shows in [7] that each squarefree monomial ideal $I$ that has a 2-linear free resolution admits a cellular minimal free resolution that is given by a regular cell complex, provided the graph to which $I$ corresponds does not contain a certain subgraph $G^{\prime}$. Though we consider only a subset of the monomial ideals with regularity two, our results for these are more explicit. In particular, we give a geometric description of the underlying polyhedral cell complexes. We also show that our results apply to the exceptional graph $G^{\prime}$ (see Example 4.3).

\section{Preliminaries}

A Ferrers graph is a bipartite graph on two distinct vertex sets $\mathbf{X}=\left\{x_{1}, \ldots, x_{n}\right\}$ and $\mathbf{Y}=\left\{y_{1}, \ldots, y_{m}\right\}$ such that if $\left(x_{i}, y_{j}\right)$ is an edge of $G$, then so is $\left(x_{p}, y_{q}\right)$ for $1 \leq p \leq i$ and $1 \leq q \leq j$. In addition, $\left(x_{1}, y_{m}\right)$ and $\left(x_{n}, y_{1}\right)$ are required to be edges of $G$. For any Ferrers graph $G$ there is an associated sequence of non-negative integers $\lambda=\left(\lambda_{1}, \lambda_{2}, \ldots, \lambda_{n}\right)$, where $\lambda_{i}$ is the degree of the vertex $x_{i}$. Notice that the defining properties of a Ferrers graph imply that $\lambda_{1}=m \geq \lambda_{2} \geq \cdots \geq \lambda_{n} \geq 1$; thus $\lambda$ is a partition. Alternatively, we can associate to a Ferrers graph a diagram $\mathbf{T}_{\lambda}$, dubbed a Ferrers tableau, consisting of an array of $n$ rows of cells with $\lambda_{i}$ adjacent cells, left justified, in the $i$-th row. A Ferrers ideal is the edge ideal associated with a Ferrers graph. Throughout this article $\lambda=\left(\lambda_{1}, \lambda_{2}, \ldots, \lambda_{n}\right)$ will always denote a fixed partition associated to a Ferrers graph $G_{\lambda}$ with corresponding Ferrers ideal $I_{\lambda}$. See [4] and [12] for additional details.

In this paper we study ideals that are closely related to Ferrers ideals. In order to explicitly describe their minimal free resolutions, we use the theory of cellular resolutions and polyhedral cell complexes as developed in [3] and [2]. We briefly recall some basic notions. However, we refer to [2] (or [11]) for a more detailed introduction.

A polyhedral cell complex $X$ is a finite collection of convex polytopes (in some $\mathbb{R}^{N}$ ) called faces (or cells) of $X$ such that:

(1) if $P \in X$ and $F$ is a face of $P$, then $F \in X$; 
(2) if $P, Q \in X$ then $P \cap Q$ is a face of both $P$ and $Q$.

Let $F_{k}(X)$ be the set of $k$-dimensional faces. Each cell complex admits an incidence function $\varepsilon$ on $X$, where $\varepsilon(Q, P) \in\{1,-1\}$ if $Q$ is a facet of $P \in X$.X is called a labeled cell complex if each vertex $i$ has a vector $\mathbf{a}_{i} \in \mathbb{N}^{N}$ (or the monomial $\mathbf{z}^{\mathbf{a}_{i}}$, where $\mathbf{z}^{\mathbf{a}_{i}}$ denotes a monomial in the variables $\left.z_{1}, \ldots, z_{N}\right)$ as label. The label of an arbitrary face $Q$ of $X$ is the exponent $\mathbf{a}_{Q}$, where $\mathbf{z}^{\mathbf{a}_{Q}}:=\operatorname{lcm}\left(\mathbf{z}^{\mathbf{a}_{i}} \mid i \in Q\right)$. Each labeled cell complex determines a complex of free $R$-modules, where $R$ is the polynomial ring $K\left[z_{1}, \ldots, z_{N}\right]$. The cellular complex $\mathcal{F}_{X}$ supported on $X$ is the complex of free $\mathbb{Z}^{N}$-graded $R$-modules

$$
\mathcal{F}_{X}: \quad 0 \rightarrow R^{F_{d}(X)} \stackrel{\partial_{d}}{\longrightarrow} R^{F_{d-1}(X)} \stackrel{\partial_{d-1}}{\longrightarrow} \cdots \stackrel{\partial_{2}}{\longrightarrow} R^{F_{1}(X)} \stackrel{\partial_{1}}{\longrightarrow} R^{F_{0}(X)} \stackrel{\partial_{0}}{\longrightarrow} R \rightarrow 0,
$$

where $d=\operatorname{dim} X$ and $R^{F_{k}(X)}:=\bigoplus_{P \in F_{k}(X)} R\left[-\mathbf{a}_{P}\right]$. The map $\partial_{k}$ is defined by

$$
\partial_{k}\left(e_{P}\right):=\sum_{Q \text { facet of } P} \varepsilon(P, Q) \cdot \mathbf{z}^{\mathbf{a}_{P}-\mathbf{a}_{Q}} \cdot e_{Q},
$$

where $\left\{e_{P} \mid P \in F_{k}(X)\right\}$ is a basis of $R^{F_{k}(X)}, e_{\emptyset}:=1$, and $\mathbf{a}_{\emptyset}:=\mathbf{0}$. If $\mathcal{F}_{X}$ is acyclic, then it provides a free $\mathbb{Z}^{N}$-graded resolution of the image $I$ of $\partial_{0}$, that is, the ideal generated by the labels of the vertices of $X$. In this case, $\mathcal{F}_{X}$ is called a cellular resolution of $I$.

Example 2.1 Consider the ideal $I:=\left(x_{1}, \ldots, x_{n}\right)\left(y_{1}, \ldots, y_{m}\right) \subset R=K\left[x_{1}, \ldots, x_{n}\right.$, $\left.y_{1}, \ldots, y_{m}\right]$. Let $X_{n, m}$ be the face complex of the polytope $\Delta_{n-1} \times \Delta_{m-1}$ obtained by taking the Cartesian product of the $(n-1)$-simplex $\Delta_{n-1}$ and the $(m-1)$ simplex $\Delta_{m-1}$. Labeling the vertices of $\Delta_{n-1}$ by $x_{1}, \ldots, x_{n}$ and the ones of $\Delta_{m-1}$ by $y_{1}, \ldots, y_{m}$, the vertices of the cell complex $X_{n, m}$ are naturally labeled by the monomials $x_{i} y_{j}$ with $1 \leq i \leq n$ and $1 \leq j \leq m$. This turns $X_{n, m}$ into a labeled polyhedral cell complex. The picture below illustrates the case where $n=2$ and $m=3$. It is shown in [4] that the complex $\mathcal{F}_{X_{n, m}}$ is a minimal free resolution of $I$. We remark that this is nothing but the tensor product of two Koszul complexes.

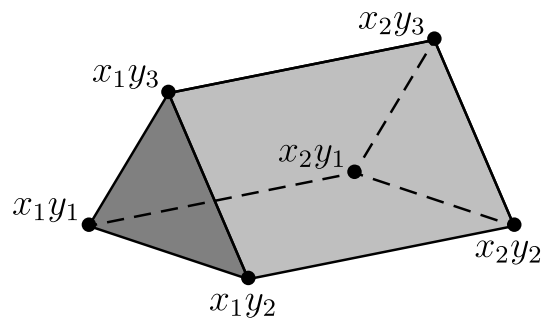

The acyclicity of $\mathcal{F}_{X}$ is merely determined by the geometry of the polyhedral cell complex $X$. Recall that $X$ is called acyclic if it is either empty or has zero reduced 
homology. Moreover, consider the partial order on $\mathbb{N}_{0}^{N}$ defined by $\mathbf{a} \preccurlyeq \mathbf{c}$ if $\mathbf{c}-\mathbf{a} \in \mathbb{N}_{0}^{N}$. For any $\mathbf{c} \in \mathbb{Z}^{N}$, we define the subcomplex $X \preccurlyeq \mathbf{c}$ of $X$ as the labeled complex that consists of the faces of $X$ whose labeling monomials $z^{\mathbf{a}}$ satisfy $\mathbf{a} \preccurlyeq \mathbf{c}$.

Bayer and Sturmfels have established the following criterion [2, Proposition 1.2] that we will use in the following section.

Lemma 2.2 The complex $\mathcal{F}_{X}$ is a cellular resolution if and only if, for each $\mathbf{c} \in \mathbb{N}_{0}^{N}$, the complex $X_{\preccurlyeq}$ is acyclic over the field $K$.

\section{Specializations}

There are relatively few monomial ideals for which the minimal free resolution is explicitly known. These include the edge ideals of bipartite graphs that are 2-regular. Up to isomorphisms these are exactly the Ferrers ideals whose minimal free resolutions have been described in [4]. Here we want to show that this information can be used to obtain the minimal free resolution of other monomial ideals by a process that we call specialization. This resolution will be again cellular.

Definition 3.1 Let I be a monomial ideal contained in $R=K\left[x_{1}, \ldots, x_{n}, y_{1}, \ldots, y_{m}\right]$. Let $\sigma:\left\{y_{1}, \ldots, y_{m}\right\} \longrightarrow\left\{x_{1}, \ldots, x_{k}\right\}$ be any map, where $k=\max \{m, n\}$ and $x_{n+1}, \ldots, x_{k}$ are (possibly) additional variables. By abuse of notation we use the same symbol to denote the substitution homomorphism $\sigma: R \longrightarrow S$, where $S:=$ $K\left[x_{1}, \ldots, x_{k}\right]$, given by $x_{i} \mapsto x_{i}$ and $y_{i} \mapsto \sigma\left(x_{i}\right)$. We call $\sigma$ the specialization map and the monomial ideal $\bar{I}:=\sigma(I) \subset S$ the specialization of $I$.

In general, the ideals $I$ and $\bar{I}$ have quite different properties.

Example 3.2 (i) Let $\lambda:=(2,2)$ and consider the specialization $\sigma$ defined by $y_{i} \mapsto x_{i}$. Then the Ferrers ideal $I_{\lambda}$ has 4 minimal generators while $\bar{I}_{\lambda}$ has only 3 minimal generators.

(ii) Consider the ideal $I=\left(x_{1} y_{1}, x_{1} y_{3}, x_{2} y_{1}\right)$ and the specialization $\sigma\left(y_{i}\right)=x_{i}$. Then $I$ and its specialization $\bar{I}$ have the same number of minimal generators, but $I$ has height two whereas $\bar{I}$ has height one. However, if we use the specialization defined by $y_{i} \mapsto x_{4-i}$, then $I$ and the specialized ideal have the same $\mathbb{Z}$-graded Betti numbers.

These examples illustrate that we need some assumptions and a careful choice of the specialization in order to study the specialized ideal by means of the original one. Throughout the remainder of this article we make the following assumption:

Assumption 3.3 Let us assume that $m \geq n$ and that $\sigma: R \longrightarrow S:=K\left[x_{1}, \ldots, x_{m}\right]$ is the specialization map defined by

$$
\sigma\left(y_{i}\right)=x_{i}
$$


In order to increase the range of graphs obtained as a specialization of Ferrers graphs, we introduce some notation for ideals that are isomorphic to Ferrers ideals.

Definition 3.4 Let $\lambda=\left(\lambda_{1}, \ldots, \lambda_{n}\right)$ be a partition and let $\mu=\left(\mu_{1}, \ldots, \mu_{n}\right) \in \mathbb{Z}^{n}$ be a vector such that

$$
0 \leq \mu_{1} \leq \cdots \leq \mu_{n}<\lambda_{n}
$$

Then we define the ideal

$$
I_{\lambda-\mu}:=\left(x_{i} y_{j} \mid 1 \leq i \leq n, \mu_{i}<j \leq \lambda_{i}\right) \subset I_{\lambda}
$$

and call it a generalized Ferrers ideal.

Note that the assumption $\mu_{n}<\lambda_{n}$ is essentially not a restriction. It just ensures that the variable $x_{n}$ divides one of the minimal generators of $I_{\lambda-\mu}$.

As in the case of Ferrers ideals, generalized Ferrers ideals correspond to a shape $\mathbf{T}_{\lambda-\mu}$ that is obtained from the Ferrers diagram $\mathbf{T}_{\lambda}$ by removing the first $\mu_{i}$ boxes in row $i$ beginning on the left-hand side. We use the notation $\lambda-\mu$ in order to distinguish it from the common notation for skew shapes. Two examples are illustrated below:

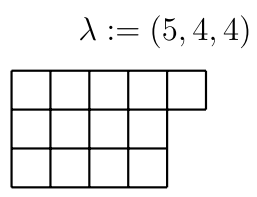

$\mathrm{T}_{\lambda}$

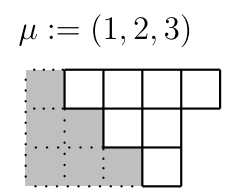

$\mathbf{T}_{\lambda-\mu}$

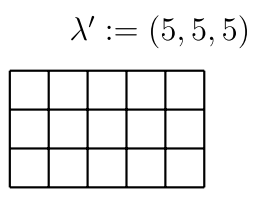

$\mathbf{T}_{\lambda^{\prime}}$

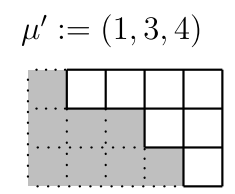

$\mathbf{T}_{\lambda^{\prime}-\mu^{\prime}}$

By reordering the columns of $\mathbf{T}_{\lambda-\mu}$ according to their length we see that $I_{\lambda-\mu}$ is isomorphic to the Ferrers ideal associated to the partition $\left(\lambda_{1}-\mu_{1}, \ldots, \lambda_{n}-\mu_{n}\right)$. Note, however, that isomorphic generalized Ferrers ideals have in general non-isomorphic specializations.

Example 3.5 Let $\lambda:=(5,4,4), \mu:=(1,2,3)$ and $\lambda^{\prime}:=(5,5,5), \mu^{\prime}:=(1,3,4)$. Then the generalized Ferrers ideals

$$
\begin{aligned}
I_{\lambda-\mu} & =\left(x_{1} y_{2}, x_{1} y_{3}, x_{1} y_{4}, x_{1} y_{5}, x_{2} y_{3}, x_{2} y_{4}, x_{3} y_{4}\right), \\
I_{\lambda^{\prime}-\mu^{\prime}} & =\left(x_{1} y_{2}, x_{1} y_{3}, x_{1} y_{4}, x_{1} y_{5}, x_{2} y_{4}, x_{2} y_{5}, x_{3} y_{5}\right)
\end{aligned}
$$

are isomorphic, while their specializations

$$
\begin{aligned}
\bar{I}_{\lambda-\mu} & =\left(x_{1} x_{2}, x_{1} x_{3}, x_{1} x_{4}, x_{1} x_{5}, x_{2} x_{3}, x_{2} x_{4}, x_{3} x_{4}\right), \\
\bar{I}_{\lambda^{\prime}-\mu^{\prime}} & =\left(x_{1} x_{2}, x_{1} x_{3}, x_{1} x_{4}, x_{1} x_{5}, x_{2} x_{4}, x_{2} x_{5}, x_{3} x_{5}\right)
\end{aligned}
$$

are not isomorphic. Indeed, the graph $\bar{G}_{\lambda^{\prime}-\mu^{\prime}}$ corresponding to $\bar{I}_{\lambda^{\prime}-\mu^{\prime}}$ has two vertices of degree two whereas the graph $\bar{G}_{\lambda-\mu}$ corresponding to $\bar{I}_{\lambda-\mu}$ does not have any vertex of degree two. 

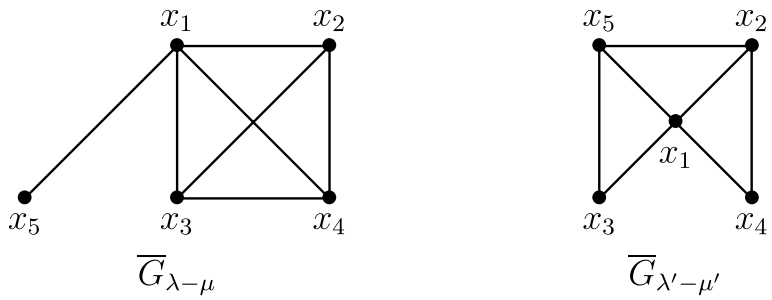

We want to show that the specialization of a generalized Ferrers ideal has a minimal free cellular resolution. This requires some preparation. We begin by describing the minimal free resolution of a generalized Ferrers ideal. The complex $X_{n, m}$ has been introduced in Example 2.1.

Definition 3.6 The labeled polyhedral cell complex $X_{\lambda-\mu}$ associated to $\lambda$ and $\mu$ is the labeled subcomplex of $X_{n, m}$ consisting of all the faces of $X_{n, m}$ whose vertices are labeled by monomials in the generalized Ferrers ideal $I_{\lambda-\mu}$.

This complex captures the information about the resolution of $I_{\lambda-\mu}$. In fact, we have:

Lemma 3.7 The complex $\mathcal{F}_{X_{\lambda-\mu}}$ offree $R$-modules provides the minimal free $\mathbb{Z}^{m+n}$ graded resolution of $I_{\lambda-\mu}$.

Proof Since the generalized Ferrers ideal is isomorphic to a Ferrers ideal, we may restrict ourselves to this case, i.e. $\mu=0$, by permuting the variables $y_{1}, \ldots, y_{m}$ suitably. For Ferrers ideals, the claim is shown as in [4, Theorem 3.2].

Corollary 3.8 The minimal $\mathbb{Z}$-graded free resolution of $I_{\lambda-\mu}$ is 2-linear, and, for $i>0$, the $i$-th Betti number of $R / I_{\lambda-\mu}$ is given by

$\beta_{i}\left(R / I_{\lambda-\mu}\right)=\left(\begin{array}{c}\lambda_{1}-\mu_{1} \\ i\end{array}\right)+\left(\begin{array}{c}\lambda_{2}-\mu_{2}+1 \\ i\end{array}\right)+\cdots+\left(\begin{array}{c}\lambda_{n}-\mu_{n}+n-1 \\ i\end{array}\right)-\left(\begin{array}{c}n \\ i+1\end{array}\right)$.

Proof This follows as in [4] because each $i$-dimensional face of $X_{\lambda-\mu}$ has a label of total degree $i+2$.

Now we want to specialize. Notice that if $\mu_{i} \leq i-2$ for some $i \geq 2$ and $\lambda_{i-1} \geq i$, the two monomials $x_{i-1} y_{i}$ and $x_{i} y_{i-1}$ in $I_{\lambda-\mu}$ specialize to the same monomial. Excluding this case, we get:

Lemma 3.9 Suppose in addition that $\mu_{i} \geq i-1, i=1, \ldots, n$. Then the ideals $I_{\lambda-\mu}$ and $\bar{I}_{\lambda-\mu}$ have the same number of minimal generators, namely $|\lambda|-|\mu|=\lambda_{1}+\cdots+$ $\lambda_{n}-\left(\mu_{1}+\cdots+\mu_{n}\right)$.

Proof The assumption guarantees that the specialization map is injective on the set of minimal generators of $I_{\lambda-\mu}$. 
This observation shows that the labels of the following complex are pairwise distinct.

Definition 3.10 The labeled polyhedral cell complex $\bar{X}_{\lambda-\mu}$ associated to $\lambda$ and $\mu$ is the complex obtained from $X_{\lambda-\mu}$ by specializing its labels. In particular, both complexes have the same supporting cell complex.

Example 3.11 Let $\lambda:=(4,4,4)$ and $\mu:=(1,2,3)$. Below we depict the complex $X_{\lambda-\mu}$ on the left-hand side and its specialization $\bar{X}_{\lambda-\mu}$ on the right-hand side.
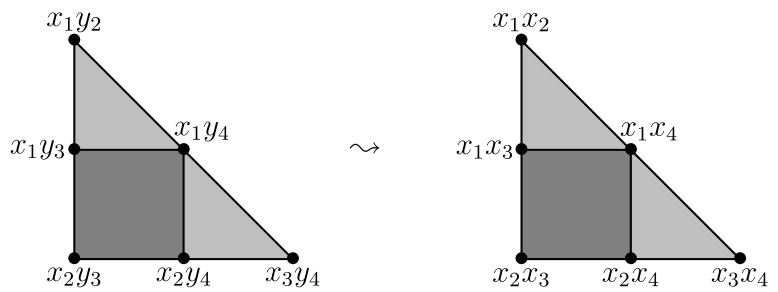

The facets of both complexes are two triangles and one rectangle.

The main result of this note is:

Theorem 3.12 If $\mu_{i} \geq i-1(i=1, \ldots, n)$, then the complex $\mathcal{F}_{\bar{X}_{\lambda-\mu}}$ of free $S$-modules provides the minimal free $\mathbb{Z}^{m}$-graded resolution of the specialization of the generalized Ferrers ideal $\bar{I}_{\lambda-\mu}$.

Proof Our strategy is to reduce the claim to the corresponding statement for generalized Ferrers ideals by applying the criterion of Bayer and Sturmfels (see Lemma 2.2) twice.

We begin with a general observation. Each face $F$ of $X_{\lambda-\mu}$ is the product of the convex hull of a set $\left\{x_{i_{1}}, \ldots, x_{i_{p}}\right\}$ and of a set $\left\{y_{j_{1}}, \ldots, y_{j_{q}}\right\}$, where $1 \leq i_{1}<\cdots<$ $i_{p} \leq n$ and $1 \leq j_{1}<\cdots<j_{q} \leq m$. The face $F$ has $p q$ vertices with labels $x_{i_{k}} y_{j_{l}}$. In particular, $x_{i_{p}} y_{j_{1}}$ is a generator of $I_{\lambda-\mu}$. Thus, the assumption $\mu_{i} \geq i-1$ implies that $i_{p} \leq j_{1}$. It follows that the label $m_{F}=x_{i_{1}} \cdots x_{i_{p}} y_{j_{1}} \cdots y_{j_{q}}$ of $F$ specializes to $x_{i_{1}} \cdots x_{i_{p}} x_{j_{1}} \cdots x_{j_{q}}$, which is equal to the least common multiple of the specializations $x_{i_{k}} x_{j_{l}}$ of the monomials labeling the vertices of $F$.

Now let $\overline{\mathbf{c}} \in \mathbb{N}_{0}^{m}$ and consider the vertices in $\left(\bar{X}_{\lambda-\mu}\right) \preccurlyeq \overline{\mathbf{c}}$. By Lemma 3.9, each such vertex corresponds to a vertex in $X_{\lambda-\mu}$ though the labels are different. Define $\mathbf{c}=$ $\left(a_{1}, \ldots, a_{n}, b_{1}, \ldots, b_{m}\right) \in \mathbb{Z}^{n+m}$ to be the unique $(n+m)$-tuple such that $x^{\mathbf{a}} y^{\mathbf{b}}$ is the least common multiple of the labels of the vertices in $X_{\lambda-\mu}$ that correspond to vertices in $\left(\bar{X}_{\lambda-\mu}\right)_{\preccurlyeq \bar{c}}$. The crucial observation is:

Claim: $\left(\bar{X}_{\lambda-\mu}\right)_{\preccurlyeq \bar{c}}$ is the labeled cell complex obtained from $\left(X_{\lambda-\mu}\right)_{\preccurlyeq \mathbf{c}}$ by specializing its labels.

To see this, let us write $x^{\mathbf{e}} \preccurlyeq x^{\mathbf{f}}$ if $\mathbf{e} \preccurlyeq \mathbf{f}$. 
Now let $F$ be a face of $\left(X_{\lambda-\mu}\right)_{\preccurlyeq \mathbf{c}}$. By the definition of $\mathbf{c}$, the labels of the vertices of $F$ specialize to monomials $x_{i_{k}} x_{j_{l}} \preccurlyeq x \overline{\mathbf{c}}$. The least common multiple of these monomials, say $x^{\mathbf{a}}$, also satisfies $x^{\mathbf{a}} \preccurlyeq x^{\mathbf{c}}$. By the above observation $x^{\mathbf{a}}$ is the specialization of the label $m_{F}$. Hence, $F$ corresponds to a face of $\left(\bar{X}_{\lambda-\mu}\right)_{\preccurlyeq \overline{\mathbf{c}}}$.

Conversely, let $\bar{F}$ be a face of $\left(\bar{X}_{\lambda-\mu}\right)_{\preccurlyeq \bar{c}}$. By the definition of $\mathbf{c}$, the vertices of $\bar{F}$ correspond to vertices of $\left(X_{\lambda-\mu}\right)_{\preccurlyeq \mathbf{c}}$. Let $x^{\mathbf{a}} y^{\mathbf{b}}$ be the least common multiple of the monomials $x_{i_{k}} y_{j_{l}}$ labeling these vertices. Then the $(n+m)$-tuple $(\mathbf{a}, \mathbf{b})$ obtained by concatenating satisfies $(\mathbf{a}, \mathbf{b}) \preccurlyeq \mathbf{c}$. Hence $\bar{F}$ corresponds to a face of $\left(X_{\lambda-\mu}\right)_{\preccurlyeq \mathbf{c}}$, and the proof of the claim is complete.

According to Lemma 3.7, the complex $\mathcal{F}_{X_{\lambda-\mu}}$ is exact. Thus Lemma 2.2 yields that the complex $\left(X_{\lambda-\mu}\right)_{\preccurlyeq \mathbf{c}}$ is acyclic over $K$. Hence, the above claim shows that $\left(\bar{X}_{\lambda-\mu}\right)_{\preccurlyeq \overline{\mathbf{c}}}$ is also acyclic. Now apply the Bayer-Sturmfels criterion (Lemma 2.2) to $\mathcal{F}_{\bar{X}_{\lambda-\mu}}$ and the proof is complete.

Corollary 3.13 If $\mu_{i} \geq i-1(i=1, \ldots, n)$, then the ideal $\bar{\Lambda}_{\lambda-\mu}$ has a 2-linear $\mathbb{Z}$ graded free resolution, i.e. its Castelnuovo-Mumford regularity is two.

Proof This follows by combining Corollary 3.8 and the claim in the above proof.

Using [5, Proposition 0.3], the last result implies in particular that $I_{\lambda-\mu}$ is the homogeneous ideal of a small subscheme in $\mathbb{P}^{m-1}$ that is not necessarily reduced. In [5, Theorem 6.1], Eisenbud, Green, Hulek, and Popescu construct a free resolution for every reduced subscheme $X$ that is the union of linear subspaces and that has a 2-linear free resolution. However, in general this resolution is not minimal though it gives the exact number of minimal generators of the homogeneous ideal $I_{X}$ of $X$. Our Theorem 3.12 treats cases where $I_{X}$ is a not necessarily reduced monomial ideal, and it has a stronger conclusion.

We can also interpret the above results using the concept of lifting. Indeed, let $I$ be an ideal in the commutative ring $A$ and let $u_{1}, \ldots, u_{t}$ be elements in $A$. Set $B:=A /\left(u_{1}, \ldots, u_{t}\right) A$ and let $J \subset B$ be an ideal. Then $I$ is said to be a $t$-lifting of $J$ if $\left\{u_{1}, \ldots, u_{t}\right\}$ is an $A / I$-regular sequence and $\left(I, u_{1}, \ldots, u_{t}\right) /\left(u_{1}, \ldots, u_{t}\right) \cong J$ (see [10, Definitions 2.1 and 2.3]).

Recall our assumption $m \geq n$. Hence $R$ is a subring of the polynomial ring $R^{\prime}:=$ $K\left[x_{1}, \ldots, x_{m}, y_{1}, \ldots, y_{m}\right]$.

Corollary 3.14 If $\mu_{i} \geq i-1(i=1, \ldots, n)$, then the ideal $I_{\lambda-\mu} R^{\prime}$ is an m-lifting of the ideal $\bar{I}_{\lambda-\mu} \subset S$.

Proof Obviously, we have $\left(I_{\lambda-\mu} R^{\prime}+\left(y_{1}-x_{1}, \ldots, y_{m}-x_{m}\right)\right) /\left(y_{1}-x_{1}, \ldots, y_{m}-\right.$ $\left.x_{m}\right) \cong \bar{I}_{\lambda-\mu}$. It remains to show that $\left\{y_{1}-x_{1}, \ldots, y_{m}-x_{m}\right\}$ is an $R^{\prime} / I_{\lambda-\mu} R^{\prime}$ regular sequence. The minimal free resolution of $I_{\lambda-\mu}$ over $R$ has the same length as the minimal free resolution of $I_{\lambda-\mu} R^{\prime}$ over $R^{\prime}$. Moreover, Theorem 3.12 shows that both resolutions have the same length as the minimal free resolution of $\bar{I}_{\lambda-\mu}$ over $S$. Hence, the Auslander-Buchsbaum formula provides that depth $R^{\prime} / I_{\lambda-\mu} R^{\prime}=$ 
$m+$ depth $S / \bar{I}_{\lambda-\mu}=m+$ depth $R^{\prime} /\left(I_{\lambda-\mu} R^{\prime}+\left(y_{1}-x_{1}, \ldots, y_{m}-x_{m}\right)\right)$. It follows that $\left\{y_{1}-x_{1}, \ldots, y_{m}-x_{m}\right\}$ is an $R^{\prime} / I_{\lambda-\mu} R^{\prime}$-regular sequence.

Probably, the last result could be shown directly by brute force, thus giving an alternative approach to the results about the resolutions of the specializations. However, our above approach seems more elegant and transparent.

\section{Threshold graphs and stable ideals}

We are going to discuss the graphs and ideals, respectively, that we obtain as specializations of Ferrers graphs and ideals. Allowing loops, each graph $G$ on the vertex set $[m]=\{1, \ldots, m\}$ defines the edge ideal $I_{G} \subset S$ that is generated by the monomials $x_{i} x_{j}$ such that $(i, j)$ is an edge of $G$. This provides a one-to-one correspondence between graphs on $[m]$ and monomial ideals in $S$ whose minimal generators all have degree two.

Consider now a graph $G$ on $[m]$ without isolated vertices. This assumption is harmless as far as the edge ideal is concerned. Order the vertices of $G$ as follows. Let 1 denote one of the vertices of highest degree. Assume we have chosen vertices $1, \ldots, i-1$ where $2 \leq i \leq m$. Then let $i$ denote one of the vertices of highest degree of the subgraph of $G$ on the vertex set $\{i, \ldots, m\}$. Now we define

$$
n:=\max \{i \mid \text { There is a vertex } j \geq i \text { such that }(i, j) \text { is an edge of } G\} \text {. }
$$

Furthermore, we set $\lambda:=\left(\lambda_{1}, \ldots, \lambda_{n}\right)$ where

$$
\lambda_{i}:=\max \{j \mid(i, j) \text { is an edge of } G\} .
$$

Note that $\lambda_{n} \geq n$ by the definition of $n$. Assume that $\lambda_{1}=m$ and that $\lambda_{1} \geq \lambda_{2} \geq \cdots$ $\geq \lambda_{n}$. Then we can define $\mu:=\left(\mu_{1}, \ldots, \mu_{n}\right)$ where

$$
\mu_{i}:=-1+\min \{j \geq i \mid(i, j) \text { is an edge of } G\} .
$$

Example 4.1 Let $G$ be the graph obtained from the complete graph on 4 vertices by taking away one edge. Then the above procedure gives $I_{G}=\bar{I}_{\lambda-\mu}$, where $\lambda=$ $(4,4), \mu=(1,2)$.
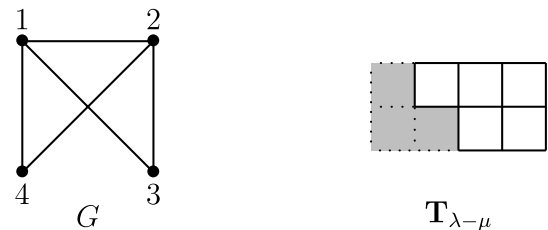

$\mathbf{T}_{\lambda-\mu}$ 
Proposition 4.2 Adopt the above notation and assume that the graph $G$ satisfies

$$
\mu_{1} \leq \mu_{2} \leq \cdots \leq \mu_{n}
$$

and

$$
(i, j) \text { is an edge of } G \text { whenever } 1 \leq i \leq n \text { and } \mu_{i}<j \leq \lambda_{i} .
$$

Then its edge ideal is $I_{G}=\bar{I}_{\lambda-\mu}$ and $\mathcal{F}_{\bar{X}_{\lambda-\mu}}$ is the minimal free cellular $\mathbb{Z}^{m}$-graded resolution of $I_{G}$. In particular,

$$
\begin{aligned}
\operatorname{reg}\left(I_{G}\right) & =2, \\
\operatorname{ht} I_{G} & =\min \left\{\min _{j}\left\{\lambda_{j}-\mu_{j}+j-1\right\}, n\right\}, \\
\operatorname{depth} S / I_{G} & =m-\max _{j}\left\{\lambda_{j}-\mu_{j}+j-1\right\}
\end{aligned}
$$

and the $i$-th Betti number of $S / I_{G}$ is given by

$$
\beta_{i}\left(S / I_{G}\right)=\left(\begin{array}{c}
\lambda_{1}-\mu_{1} \\
i
\end{array}\right)+\left(\begin{array}{c}
\lambda_{2}-\mu_{2}+1 \\
i
\end{array}\right)+\cdots+\left(\begin{array}{c}
\lambda_{n}-\mu_{n}+n-1 \\
i
\end{array}\right)-\left(\begin{array}{c}
n \\
i+1
\end{array}\right) .
$$

Proof This follows by Theorem 3.12 and Corollary 3.14 from the corresponding results for Ferrers ideals in [4].

Example 4.3 Let $G^{\prime}$ be the graph obtained from the complete graph on 4 vertices by taking away two edges that share a common vertex. Then the above procedure shows that Proposition 4.2 applies to $G^{\prime}$ with $n=2$ and $\lambda=(4,3), \mu=(1,2)$.
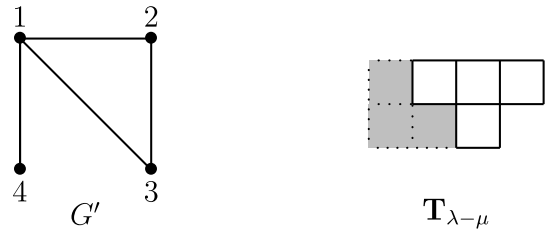

Notice that $G^{\prime}$ with a particular labeling is the graph that is excluded as a pattern of the graphs considered in [7].

Corollary 4.4 Adopt the notation and assumptions of Proposition 4.2. Then $S / I_{G}$ is a Cohen-Macaulay ring if and only if

$$
\min _{j}\left\{\lambda_{j}-\mu_{j}+j-1\right\}=\max _{j}\left\{\lambda_{j}-\mu_{j}+j-1\right\} \leq n .
$$

Proof $S / I_{G}$ is Cohen-Macaulay if and only $\operatorname{dim} S / I_{G}=\operatorname{depth} S / I_{G}$. Hence Proposition 4.2 provides the claim. 
We now discuss classes of graphs or ideals to which Proposition 4.2 applies. Recall that a monomial ideal $I \subset S$ is called strongly stable if $x_{i} \frac{x^{\mathbf{a}}}{x_{j}} \in I$ whenever $x^{\mathbf{a}} \in I$, $x_{j}$ divides $x^{\mathbf{a}}$, and $1 \leq i<j$. A squarefree monomial ideal $I$ is said to be squarefree strongly stable if $x_{i} \frac{x^{\mathbf{a}}}{x_{j}} \in I$ whenever $x^{\mathbf{a}}$ is a minimal generator of $I, x_{j}$ divides $x^{\mathbf{a}}, x_{i}$ does not divide $x^{\mathbf{a}}$, and $1 \leq i<j$. Note that in general there are the weaker conditions of being stable or squarefree stable. However, for ideals generated in degree two, the corresponding concepts are equivalent. Eliahou and Kervaire describe in [6] the minimal free resolution of an arbitrary strongly stable ideal $I$. If $I$ is generated in degree two, our results show that $I$ admits a cellular resolution. More precisely:

Example 4.5 Let $I \subset S$ be a strongly stable ideal whose minimal generators have degree two and such that $x_{1} x_{m} \in I$. Let $G$ be the corresponding graph. Then the stability property guarantees that $G$ satisfies condition $(*)$ where $\mu_{i}=i-1$ for $i=1, \ldots, n$. In particular, Corollary 4.4 immediately implies the well-known fact that $S / I$ is Cohen-Macaulay if and only if $m=\lambda_{1}=\cdots=\lambda_{n}=n$, that is $I=\left(x_{1}, \ldots, x_{m}\right)^{2}$.

Recall that a graph $G$ on $[m]$ is called a threshold graph if there is a vector $w=\left(w_{1}, \ldots, w_{m}\right) \in \mathbb{R}^{m}$ such that $(i, j)$ is an edge of $G$ if and only if $w_{i}+w_{j}>0$. We refer to the book by Mahadev and Peled [9] for a wealth of information on threshold graphs and to the work of Klivans and Reiner [8] for many alternative characterizations of threshold graphs.

Corollary 4.6 Let $G$ be a threshold graph on $[m]$ and denote by $\lambda_{i}^{\prime}$ the degree of the vertex $i$. Order the vertices such that $\lambda_{1}^{\prime} \geq \lambda_{2}^{\prime} \geq \cdots \geq \lambda_{m}^{\prime} \geq 1$. Define

$$
\begin{aligned}
n & :=\max \left\{i \mid \lambda_{i}^{\prime} \geq i\right\}, \\
\lambda=\left(\lambda_{1}, \ldots, \lambda_{n}\right) & :=\left(\lambda_{1}^{\prime}+1, \ldots, \lambda_{n}^{\prime}+1\right),
\end{aligned}
$$

and

$$
\mu=\left(\mu_{1}, \ldots, \mu_{n}\right):=(1,2, \ldots, n) .
$$

Then the edge ideal of $G$ is $I_{G}=\bar{I}_{\lambda-\mu}$ and $\mathcal{F}_{\bar{X}_{\lambda-\mu}}$ is the minimal free cellular $\mathbb{Z}^{m}$ graded resolution of $I_{G}$. In particular, $\operatorname{reg}\left(I_{G}\right)=2$, ht $I_{G}=n, \operatorname{depth} S / I_{G}=1$ and the $i$-th Betti number of $S / I_{G}$ is given by

$$
\beta_{i}\left(S / I_{G}\right)=\left(\begin{array}{c}
\lambda_{1}-1 \\
i
\end{array}\right)+\left(\begin{array}{c}
\lambda_{2}-1 \\
i
\end{array}\right)+\cdots+\left(\begin{array}{c}
\lambda_{n}-1 \\
i
\end{array}\right)-\left(\begin{array}{c}
n \\
i+1
\end{array}\right) .
$$

Proof It is known (see [8]) that a graph without loops is threshold if and only if it is shifted. Equivalently, the edge ideals of threshold graphs are precisely the squarefree strongly stable ideals that are generated in degree two. It follows that each threshold graph $G$ satisfies the conditions in Proposition 4.2 with $\mu_{i}=i$ for $i=1, \ldots, n$. The tableaux $\mathbf{T}_{\lambda-\mu}$ corresponds to the so-called up-degree sequence of $G$. It is determined by the degrees of the vertices of $G$ as stated. 
We illustrate this proof with the following example.

Example 4.7 Consider the graph $G^{\prime}$ as depicted in Example 4.3. It is a threshold graph on 4 vertices whose degree sequence is $(3,2,2,1)$. Hence, using the notation of Corollary 4.6, we get $n=2, \lambda=(4,3)$, and $\mu=(1,2)$. Thus, we see again that the edge ideal of $G^{\prime}$ equals $\bar{I}_{\lambda-\mu}$.

Remark 4.8 (i) Corollary 4.6 shows explicitly how each threshold graph can be obtained as the specialization of a Ferrers graphs. More precisely, using the fact that edge ideals of threshold graphs are squarefree strongly stable [8], it describes how to obtain such an ideal as the specialization of the edge ideal of a suitable Ferrers graph. Thus, Corollary 4.6 explains the similar algebraic properties of Ferrers and threshold graphs.

(ii) The minimal free resolution of an arbitrary squarefree stable monomial ideal has been described by Aramova, Herzog and Hibi in [1]. However, it is not given as a cellular resolution.

We end our note by remarking that, by suitably modifying the vertex labels if necessary, our methods apply to more graphs than discussed so far.

Example 4.9 Consider the graph $\bar{G}_{\lambda^{\prime}-\mu^{\prime}}$ that is described in Example 3.5. This is the same graph as the one studied in [7, Example 4.3], but with a different labeling. As remarked earlier, its edge ideal is the specialization of $I_{\lambda^{\prime}-\mu^{\prime}}$ with $\lambda^{\prime}=(5,5,5)$ and $\mu^{\prime}=(1,3,4)$. Hence the cellular resolution of $\bar{G}_{\lambda^{\prime}-\mu^{\prime}}$ is given by the polyhedral cell complex pictured below

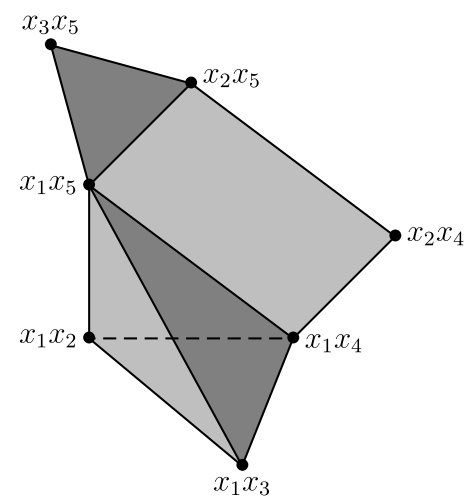

This cell complex has a 2-simplex as a facet, whereas Horwitz's cell complex does not have such a facet. This shows in particular that the maps in the free resolutions constructed by Horwitz and by our methods are in general different.

After completing the first version of this note, Horwitz informed us that by applying his methods to the graph $\bar{G}_{\lambda^{\prime}-\mu^{\prime}}$ with the above labeling, he gets the same abstract cell complex, but with a different labeling of its vertices, so again his resulting maps in the free resolution are different from ours. 
Acknowledgements The authors would like to thank Vic Reiner for inspiring discussions and the anonymous referees for their careful reading of the original manuscript.

Uwe Nagel gratefully acknowledges partial support from the NSA under grant H98230-07-1-0065.

\section{References}

1. Aramova, A., Herzog, J., Hibi, T.: Squarefree lexsegment ideals. Math. Z. 228, 353-378 (1998)

2. Bayer, D., Sturmfels, B.: Cellular resolutions of monomial modules. J. Reine Angew. Math. 502, 123-140 (1998)

3. Bayer, D., Peeva, I., Sturmfels, B.: Monomial resolutions. Math. Res. Lett. 5, 31-46 (1998)

4. Corso, A., Nagel, U.: Monomial and toric ideals associated to Ferrers graphs. Trans. Am. Math. Soc. (2007, to appear)

5. Eisenbud, D., Green, M., Hulek, K., Popescu, S.: Small schemes and varieties of minimal degree. Am. J. Math. 128, 1363-1389 (2006)

6. Eliahou, S., Kervaire, M.: Minimal resolutions of some monomial ideals. J. Algebra 129, 1-25 (1990)

7. Horwitz, N.: Linear resolutions of quadratic monomial ideals. J. Algebra 318, 981-1001 (2007)

8. Klivans, C., Reiner, V.: Shifted set families, degree sequences, and plethysm. Electron. J. Comb. (2007, to appear)

9. Mahadev, N.V.R., Peled, U.N.: Threshold Graphs and Related Topics. Annals Discrete Mathematics, vol. 56. North-Holland, Amsterdam (1995)

10. Migliore, J., Nagel, U.: Lifting monomial ideals. Commun. Algebra 28, 5679-5701 (2000). Special volume in honor of R. Hartshorne

11. Miller, E., Sturmfels, B.: Combinatorial Commutative Algebra. Graduate Texts in Mathematics, vol. 227. Springer, New York (2005)

12. Villarreal, R.: Monomial Algebras. Monographs and Textbooks in Pure and Applied Mathematics, vol. 238. Marcel Dekker, New York (2001) 